\title{
Clothing Performance during (IR) Heat Radiation Exposure
}

\author{
Uwe Reischl $^{1 *}$ and Budimir Mijovic ${ }^{2}$ \\ ${ }^{1}$ Department of Community and Environmental Health, Boise State University, Idaho, USA \\ ${ }^{2}$ Budimir Mijovic Faculty of Textile Technology, University of Zagreb, Croatia
}

*Corresponding author: Uwe Reischl, Professor, Department of Community and Environmental Health, Boise State University, Idaho, USA

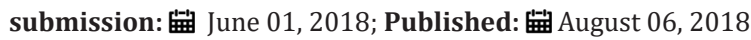

\begin{abstract}
Thermal manikin tests were performed on two protective garment types to evaluate their heat transfer properties during exposure to (IR) heat radiation. Comparing a newly developed Heat Radiation (IR) attenuation vest designed for use by agricultural workers and a standard winter jacket, it could be seen that the new IR attenuation vest provided a thermal comfort benefit above a heat radiation exposure level of 150 watts while the winter jacket provided a thermal benefit above a 450watt heat radiation (IR) exposure level. The study identified an important experimental temperature cross-over point which can be used to define the thermodynamic properties of a garment during exposure to IR heat radiation. This allows the characterization of temperature insulation properties of a garment. The evaluation method presents a new approach for predicting garment comfort and safety during various intensities of heat radiation (IR) exposure.
\end{abstract}

Keywords: Protective clothing; IR heat radiation; Comfort; Safety; Thermodynamic characteristics

\section{Introduction}

Workers exposed to outdoor solar heat radiation are at increased risk of suffering heat related disorders. Use of tents, hats, and other protective equipment are frequently used to reduce such exposures. However, such measures are often impractical if employees must change postures or are required to move frequently to new locations as part of their job performance. Innovative use of clothing materials and creative approaches to garment design can help reduce clothing heat stress during exposure to such outdoor solar environments.

While garments can offer a barrier to solar heat radiation, garments can also lead to physiological heat stress by limiting the release of body metabolic heat. The goal of this study was to identify a new approach that could be used to balance garment insulation with garment heat radiation attenuation. To pursue this objective, experiments were conducted to evaluate the thermodynamic characteristics of two garment types documenting the infrared heat radiation penetration characteristics, the fabric radiation absorption characteristics and the garment insulation.

In studying clothing induced heat stress, the human body should be seen as a heat producing system that must maintain a balance between heat loss and heat gain. Environmental parameters such as air temperature, air velocity, radiant heat, and humidity can affect this delicate balance [1-3]. Clothing material and garment design can also influence this heat balance by promoting or reducing heat exchange by sweat evaporation, convection, conduction, and heat radiation [4]. The performance of fabrics under such conditions is also linked to the chemical and physical structure of the garment materials including thickness and weight. For example, it has been shown that woven textile materials do not offer a strong barrier against penetration by infrared radiation. However, the performance is better when the fabric thickness is greater and when the fabric material is heavier [5].

\section{Measurement Platform}

An inflatable thermal manikin system was used that allowed a garment to be evaluated for convective, conductive, and radiative heat exchange. While the manikin system did not simulate human physiological responses, the technology provided a stable platform for determining the thermodynamic properties of a wide range of garment configurations.

The system is illustrated in Figure 1. The manikin's shape and dimensions corresponded to a "Medium" sized male. Heat loss and heat gain is determined by the difference between the temperature of manikin input air and the temperature of the manikin output air, i.e., $\Delta \mathrm{T}^{\circ} \mathrm{C}$. The system is calibrated and allows for a conversion from the $\Delta \mathrm{T}^{\circ} \mathrm{C}$ value to power units, i.e., Watts. This value is used as the dependent variable in the experiments. Clothing and heat radiation 
exposure conditions were designated as independent variables. A semi-nude configuration provided a data reference, i.e., a "control" for comparison purposes. Figure 2 illustrates the semi-nude "control" manikin configuration, the IR Vest configuration, and the jacket configuration.

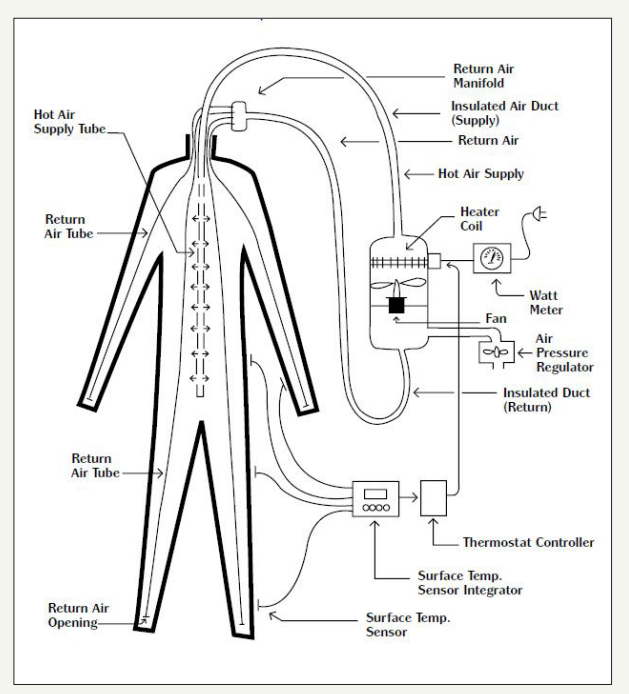

Figure 1: Thermal manikin system used in determining heat transfer characteristics of protective clothing.

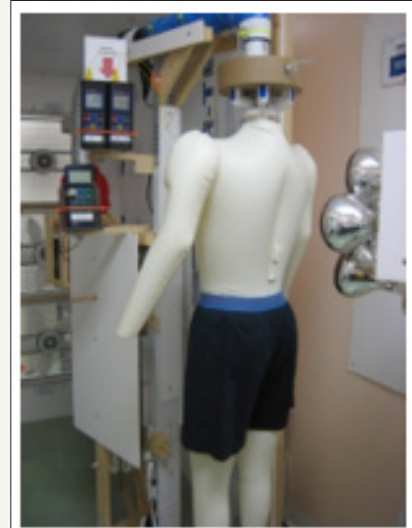

A

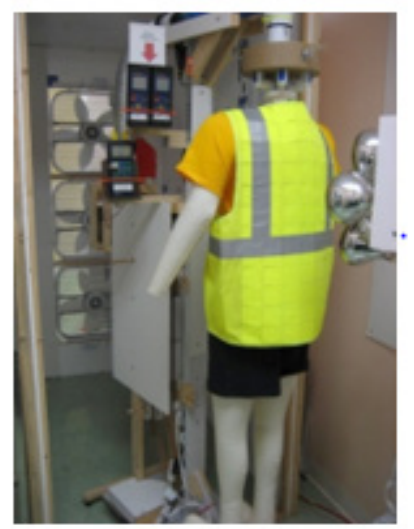

B

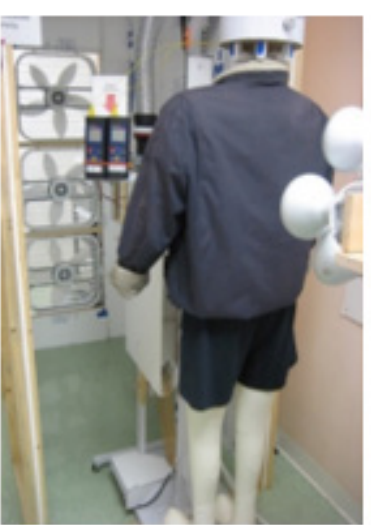

C

Figure 2: Illustration of manikin semi-nude "control" configuration (A), IR Attenuation vest (B), and winter jacket (C).

\section{Procedure}

All tests were conducted inside a temperature-controlled laboratory using a previously described protocol [6]. Clothing configurations included a new prototype infrared reflecting vest (IR Attenuation Vest) developed for use by agricultural workers in outdoor environments and a standard winter jacket. The two garments were selected because of their performance differences in attenuating infrared heat radiation. Infrared heat radiation exposure levels ranged from 0watts to 500watts in 100watt increments.

\section{Results and Analysis}

Incremental exposures to IR radiation resulted in a linear increase in the manikin's internal temperature for both clothing configurations. However, the slope (rate) of this increase was lower for the IR Attenuation Vest than for the semi-nude condition. When the manikin was clothed with the standard winter jacket, this slope was smaller than the slope for the IR vest. The slopes observed for both the IR Attenuation Vest and the standard winter jacket define the heat radiation attenuation characteristic of the garment, i.e., the lower the slope, the higher the heat radiation attenuation characteristic. However, the insulation heat gain observed for the standard winter jacket was higher than the insulation heat gain observed for the IR Attenuation Vest.

Figure 3 illustrates the relationship between manikin heat gain, heat radiation exposure, and clothing. The semi-nude manikin gained the most heat when exposed to the IR heat radiation but exhibited no measurable heat gain as a result of clothing insulation. The winter jacket exhibited the lowest heat gain due to heat radiation but exhibited the highest heat gain due to garment insulation. The IR Attenuation Vest exhibited values between the two. Total body heat loading (heat gain) for both garment types 
included two factors: Heat absorbed from the infrared radiation penetrating the garments and heat retention due to clothing insulation. It can be seen that the IR Attenuation Vest intersects the "control" graph at point " $\mathrm{A}$ " while the standard winter jacket intersects the "control" graph at point "B". These two "cross-over" points define the conditions where the radiation heat load imposed on the semi-nude manikin is equivalent to the heat load imposed on the manikin by both heat radiation and garment insulation. Garments with a "low" cross-over point can be seen as "superior" to garments with a "high" cross-over point because garments with a low cross-over point impose lower insulation heat stress while providing better protection against IR infrared heat radiation $[7,8]$.

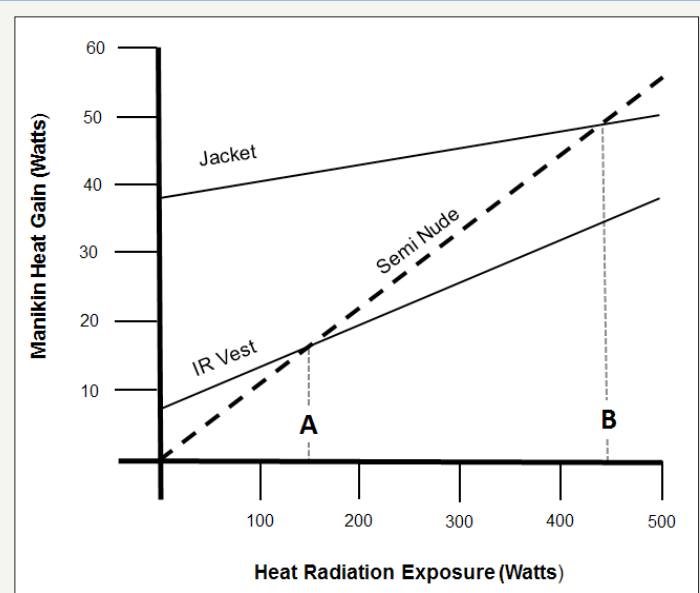

Figure 3: Manikin heat gain associated with the semi-nude configuration, IR Attenuation Vest and the winter jacket during exposure to increasing infrared heat radiation levels.

\section{Conclusion}

This study successfully documented the thermal performance of a prototype IR attenuation vest and the performance of a standard winter jacket under controlled infrared (IR) heat radiation exposure conditions by using temperature cross-over points as reference indicators of body heat loading. The cross-over points were able to identify the thermodynamic properties of both clothing ensembles. This evaluation method now offers a simple tool for not only evaluating the performance of existing garments but can also be used to optimize the comfort and safety of new garments using innovative designs and new materials.

\section{References}

1. Bishop P, Smith G, Ray P, Beaird J, Smith J (1994) Empirical prediction of physiological response to prolonged work in encapsulating protective clothing. Ergonomics 37(9): 1503-1540.

2. Montain S, Sawka M, Cadarette B, Quigley M, MacKay J (1994) Physiological tolerance to uncompensable heat stress: Effects of exercise intensity, protective clothing, and climate. J Appl Physiol 77(1): 216-222.
3. Holmer I (1995) Protective clothing and heat stress. Ergonomics 38(1): 166-182.

4. Reischl U, Colby C (2010) "Pneumotech": A new tool for evaluating the thermal characteristics of clothing systems. In Mijovic B (Edr.), Proceedings of the $4^{\text {th }}$ International Ergonomics Conference, Zagreb, Croatia, pp. 29-36.

5. Sun GY, Zhang XS, Pan N (2000) Radiant protective and transport properties of fabrics used by wildland firefighters. Textile Research Journal 70(7): 567-573.

6. Mijovic B, Skenderi Z, Camara J, Reischl U, Colby C, et al. (2009) Inflatable mannequin for testing thermal properties of clothing. Proceedings of the $17^{\text {th }}$ World Congress on Ergonomics. Beijing, IEA, China.

7. Reischl U (2013) Optimizing protective clothing design for hot outdoor environments. Advanced Engineering Forum10: 89-92.

8. Reischl U, Goonetilleke R, Mijovic B, Skenderi Z (2011) Thermal characteristics of infrared radiation protective vests. Sigurnost 53(1): 51-56.
Creative Commons Attribution 4.0

International License

For possible submissions Click Here

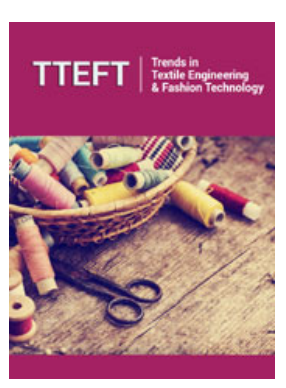

Trends in Textile Engineering \& Fashion Technology

\section{Benefits of Publishing with us}

- High-level peer review and editorial services

- Freely accessible online immediately upon publication

- Authors retain the copyright to their work

- Licensing it under a Creative Commons license

- Visibility through different online platforms 